\title{
Performed Imaging
}

National Cancer Institute

\section{Source}

National Cancer Institute. Performed Imaging. NCI Thesaurus. Code C93428.

The completed action of obtaining pictures of the interior or exterior of the body usually for diagnostic reasons. 